\title{
Quality assurance during interstitial brachytherapy: in vivo dosimetry using MOSFET dosimeters
}

\author{
Corinna Melchert, PhD, Tamer Soror, MD, Prof. György Kovács, MD, PhD \\ Interdisciplinary Brachytherapy Unit, University of Luebeck/UKSH-CL, Luebeck, Germany
}

\begin{abstract}
Purpose: Brachytherapy procedure may result in acute tissue reactions like edema, causing deviations between planned and measured doses. The rationale for in vivo dosimetry in interstitial brachytherapy is to assess the accuracy of the delivered dose in comparison with the dose calculated by the treatment planning system (TPS).

Material and methods: One single computer tomography (CT) dataset was used for brachytherapy planning, taken within 24 hours after implantation. In vivo interstitial measurements with micro-MOSFET-detectors (metal oxide semiconductor field effect transistor) were performed in 12 patients with different anatomic locations of cancers, including thorax-wall, head and neck, breast, and different types of implantations (monoplanar, loops, and multiplanar).

Results: Measured values for the thorax-wall tumor patient showed a good agreement with the calculated data, with average deviation of $-2.7 \%$ in $8 \mathrm{~mm}$ distance to the closest dwell position of the source. The deviation of the measured dose value of the head and neck patient was $+55.6 \%$ in the first fraction and $+8.5 \%$ in the last fraction. In the ten breast cancer patients, measured doses depended on the proximity of the detector to the irradiated volume PTV.

Conclusions: The deviations between planned and measured dose values were markedly influenced by the proximity of the detector to the PTV because where the edema exerts, the greatest influence on the tube applicator geometry. The positioning of the patient during irradiation must correspond to the positioning in the planning CT. Further studies are needed to investigate the role of in vivo dosimetry during interstitial brachytherapy as a routine procedure.

J Contemp Brachytherapy 2018; 10, 3: 232-237

DOI: https://doi.org/10.5114/jcb.2018.76748
\end{abstract}

Key words: MOSFET, in vivo, dosimetry, interstitial brachytherapy, quality assurance, treatment-plan verification.

\section{Purpose}

Cancer irradiation through interstitial high-dose-rate (HDR) brachytherapy includes an implantation of plastic tubes inside target area. Due to tissue manipulation during this procedure, some immediate tissue reactions may occur as inflammation and edema [1]. The size of edema depends on the extent of the damage inside the tissue.

Post-implant thin-slice computed tomography (CT) of $1 \mathrm{~mm}$ is used for the treatment planning. The length of the data set must include the entire irradiation range. The reproducibility of the slice thickness and the quality assurance (QA) of the CT scan are presented by the deviation of lengths of applicator tubes, which are measured on one hand with a special check ruler and on the other, digitized in the treatment planning system (TPS). There is a difference in length of the tubes of maximum $\pm 0.5 \mathrm{~mm}$. Generally, only one single dataset is used for dose calculation. Any deviation in the patient's anatomy compared to CT dataset used will result in changes in the actual dose distribution. Edema results in changes in the size of the planning target volume (PTV) compared to the size of PTV in the planning CT dataset. Interspacing between brachytherapy tubes may also be changed, and the actual delivered dose might be higher or lower than the calculated one. Assessment of the final uncertainties between the calculated and the actual delivered dose is an elementary need in treatment QA.

The rationale for in vivo dosimetry in interstitial brachytherapy is the real time control of delivered dose. In vivo dosimetry in brachytherapy is recommended by the International Commission of Radiological Units (ICRU) [2,3].

The threshold of the gate voltage of a metal oxide semiconductor field effect transistor (MOSFET) changes proportionally with the absorbed radiation dose. MOSFETs have proved to be useful and effective in vivo dosimeters for quality assurance in HDR brachytherapy [4].

Interstitial dose measurement with microMOSFETs is a well-suited method to identify the actual delivered dose in patients during treatment, and provides an important audit for treatment precision [5]. The readings from the microMOSFET detectors were found to be reproducible
Address for correspondence: Corinna Melchert, PhD, Interdisciplinary Brachytherapy Unit, University of Luebeck/UKSH-CL, Ratzeburger Allee 160, 23538 Luebeck, Germany, phone: +49 451500454 63, fax: +49 451500454 24, e-mail: corinna.melchert@uksh.de
Received: 12.01 .2018

Accepted: 04.06 .2018

Published: 30.06 .2018 
and slightly directionally dependent. They are suitable for in vivo dosimetry, as they require a very small electrical voltage and accordingly, there is no danger for patient in getting an electrical shock. The sensitive part of the microMOSFET detector is of small size and can be positioned inside the 6-French diameter plastic tube applicators. In this study, online in vivo dosimetry was implemented by measuring the dose inside the implanted tubes during treatment delivery.

The purpose is to present the difference between the prescribed dose as calculated by the TPS and the actual measured dose using microMOSFET detectors for in vivo dosimetry in fractionated interstitial brachytherapy.

\section{Material and methods}

The calculated dose by the TPS Oncentra (ELEKTA, Stockholm, Sweden) was compared to the measured dose (at specific points) within the implanted plastic tube applicators inside a patient. The measurements were carried out on 12 patients: 1 thorax-wall, 1 head and neck, and 10 breast, using the microMOSFET dosimeter TN-502RDM (standard), the wireless dosimetry system, and the mobileMOSFET dose verification system TN-RD-70-W (Best Medical Canada, Ottawa). One microMOSFET standard has an active volume of $0.2 \times 0.2 \times 5 \times 10^{-4} \mathrm{~mm}^{3}$ [6]. Due to this small active volume, it has a high spatial resolution and fits into 6-French plastic tube applicator. The MOSFETs run on battery power and were calibrated on standard sensitivity. The electrical voltage applied to the MOSFET detector is 7 volts. Therefore, it was suitable for measuring the accurate dose value at a distance from the ${ }^{192} \mathrm{Ir}$ source down to $5 \mathrm{~mm}$ [7]. The accuracy of measurements of radiation dose using the MOSFET detector is $\pm 4 \%$. The characteristics of the MOSFET detectors are described by the manufacturer [8]. Zilio et al. evaluated and confirmed the accuracy of the MOSFET dosimeter as an absolute dosimeter for brachytherapy [9]. The calibration procedure was performed following the recommendation of the American Association of Physicists in Medicine, AAPM-MOSFET [10].

The determination of the locations of the MOSFETS readings inside the tubes followed two assumptions. First, locations with low influence of tissue disturbances on the calculated dose should be positioned distant from the PTV. Second, locations with high influence of tissue disturbances on the calculated dose would be expected to be close to the PTV or within the PTV.

The identification of the planned measurement locations for the MOSFET detectors was performed on the TPS. This leads to a specific length of the MOSFET wire inside the tube, and the desired length of the MOSFET wire was marked with an adhesive tape. The wire was pushed up to the mark into the tube. To keep its sensitive part at the precise location, it was fixed with a second adhesive tape to the outside of the tube (see Figure 1). Uncertainty margin for positioning was estimated to be $\pm 1 \mathrm{~mm}$.

For the breast cancer patients, a comparison with the planning CT dataset recorded one day after the implantation and the radiography taken at the end of the treatment was used to find any deviations in the phys- ical representation of the anatomy. In order to spare an additional radiation exposure to a patient, no extra CT dataset was taken. The maximum intensity projection (MIP) from CT slices was calculated and both datasets were compared (applying the same scale and angles). The surgical clips (which mark the tumor bed) were used as landmarks for comparison. To prevent the slipping of the tubes from a patient, special buttons were used for fixation. The distances between them were respectively measured for both different datasets. Six distances between eleven buttons in both different recorded datasets were determined.

In the first patient, MOSFET measurements were progressively measured for a patient with a thorax-wall tumor. The treatment included 12 fractions and required only a single plane of tubes implanted parallel to the thorax-wall. Two detectors were placed in two different tubes, respectively, of the thirteen tubes in total. One detector was placed at $8 \mathrm{~mm}$, and the second at $2.8 \mathrm{~mm}$ distance to the closest dwell position of the source. Dose values were taken every second fraction. A dosimetry protocol is shown in Table 1.

In the head and neck cancer patient, five plastic tube applicators were implanted to irradiate the base of the tongue. The CT dataset was obtained immediately following the implantation procedure. Figure 1 presents the geometry of the applicators.

Fourteen fractions were delivered and in every second dose, measurements were performed and started at the third fraction. To prove the right dose on the skin, one MOSFET detector was placed on patient's chin.

The next case presents measurements from ten breast cancer patients. Tube applicators were implanted in three to four successive geometric levels. Dose measurements were obtained from different positions in relation to the PTV and during different days, earliest, on the third day after taking CT dataset (four days after implantation of the tube). The last measurement was taken for each patient at the last fraction (fraction number 8). Figure 2A shows the location of implanted tube applicators inside a breast cancer patient. This was an attempt to demonstrate that the location at the border to the PTV should not be affected very much by the presence of an edema or other disturbances inside the treated volume. Figure $2 \mathrm{~B}$ shows the locations of detectors in the outer plane of the implantation volume. Figure $2 \mathrm{C}$ shows one location distant to the PTV (upper arrow), two positioned inside the PTV (middle arrows), and one close to the PTV (lower arrow).

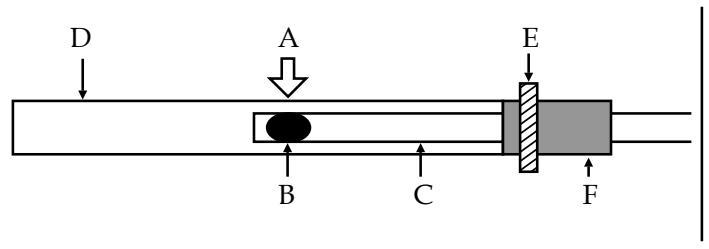

Fig. 1. Positioning the MOSFET dosimeter inside the tube. A-measuring point, B-MOSFET detector, C-MOSFET wire, $\mathrm{D}$ - tube applicator, $\mathrm{E}$ - adhesive mark, F - adhesive fixation 
Table 1. For thorax-wall irradiation, a dosimetry protocol with calculated and measured dose values as well as deviations is shown in percentage

\begin{tabular}{|c|c|c|c|c|c|c|}
\hline \multicolumn{3}{|c|}{ Tube 3} & \multirow[t]{2}{*}{ Fraction } & \multicolumn{3}{|c|}{ Tube 6} \\
\hline $\begin{array}{c}\text { Calculated dose } \\
\text { (Gy) }\end{array}$ & $\begin{array}{l}\text { Measured dose } \\
\text { (Gy) }\end{array}$ & $\begin{array}{l}\text { Deviation } \\
(\%)\end{array}$ & & $\begin{array}{l}\text { Calculated dose } \\
\text { (Gy) }\end{array}$ & $\begin{array}{c}\text { Measured dose } \\
\text { (Gy) }\end{array}$ & $\begin{array}{c}\text { Deviation } \\
(\%)\end{array}$ \\
\hline 2.97 & 2.80 & -5.7 & 2 & 5.10 & 6.32 & 23.9 \\
\hline 2.97 & 2.88 & -3.0 & 4 & 5.10 & 5.88 & 15.3 \\
\hline 2.97 & 2.84 & -4.4 & 6 & 5.10 & 5.74 & 12.5 \\
\hline 2.97 & 2.95 & -0.7 & 8 & 5.10 & 5.52 & 8.2 \\
\hline 2.97 & 2.98 & 0.3 & 10 & 5.10 & 6.13 & 20.2 \\
\hline 2.97 & 2.89 & -2.7 & 12 & 5.10 & 5.76 & 12.9 \\
\hline 2.97 & 2.89 & -2.7 & Mean & 5.10 & 5.89 & 15.5 \\
\hline
\end{tabular}

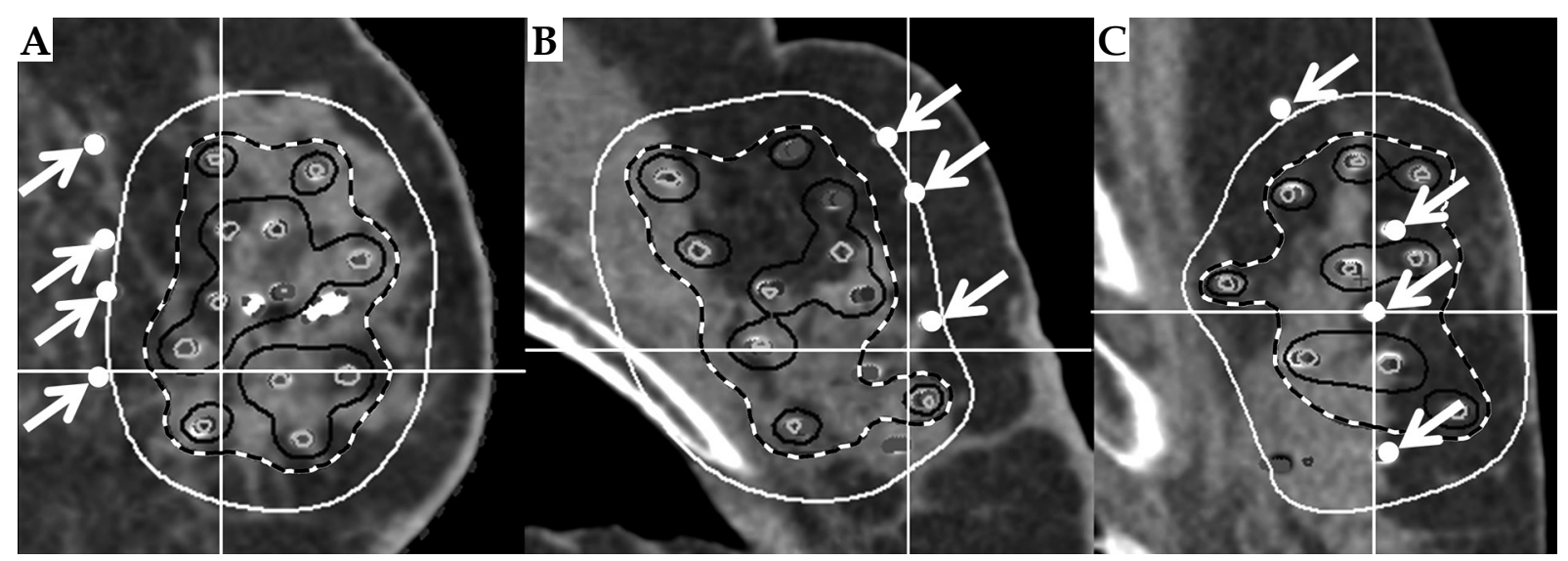

Fig. 2. Irradiation against breast cancer for different patients. Light grey: $50 \%$ isodose; black/white dashed line corresponds to the PTV $=100 \%$ isodose; black line $150 \%$ isodose; grey line 400\% isodose. Arrows show the MOSFET positions. A) Measurements in tubes positioned close to the ribs. B) Measurements in tubes close to body surface. C) Upper arrow: measurements distant to the PTV, the two middle arrows: measurement inside the PTV and the lower arrow measurements close to the PTV

\section{Results}

The estimation of the presence of a post-implant edema or any other disturbances inside the tissue related to the implantation procedure was conducted by comparing different datasets described above. In average, the outcome of the comparison demonstrated 5.37\% larger distances between two specific buttons for the CT dataset taken one day after the intervention than in the radiography taken seven days later.

\section{Thorax-wall}

The results of the microMOSFET measurements were taken in the fraction: $2,4,6,8,10$, and 12 . Dose values measured inside tube no. 3 showed a good agreement with the calculated data; the average deviation was $2.7 \pm 2 \%$ or $0.08 \pm 0.06 \mathrm{~Gy}$. The differences were smaller than the inaccuracy of the MOSFET detectors $( \pm 4 \%)$. Results represented by the measurements in tube no. 6 showed large discrepancies from the calculated data.
The mean value with 5.89 Gy was equivalent to $15 \%$ higher of the predicted dose value of $5.1 \mathrm{~Gy}$. In this case, the detector was located very close to the nearest dwell position of the source. It represented $2.8 \mathrm{~mm}$ distance from the closest dwell position and was affected by the presence of the high-dose gradient [9] region.

\section{Head and neck}

The CT dataset was captured right after the implantation procedure with a very low chance for the development of subsequent edema. The measurements were conducted seven times at the same position inside the tube demonstrated in Figure 3. This detector was $3.9 \mathrm{~mm}$ from the closest dwell position inside the PTV. The first dose measurement started in the third fraction, four days after the recording of the $\mathrm{CT}$ dataset. The calculated dose value at this point was $4.3 \mathrm{~Gy}$. The measurement reached, in contrast, a value of $6.7 \mathrm{~Gy}$, which is $55.6 \%$ larger than predicted. The results of the following measurements showed a decrease in the dose values during 12 days of treatment 

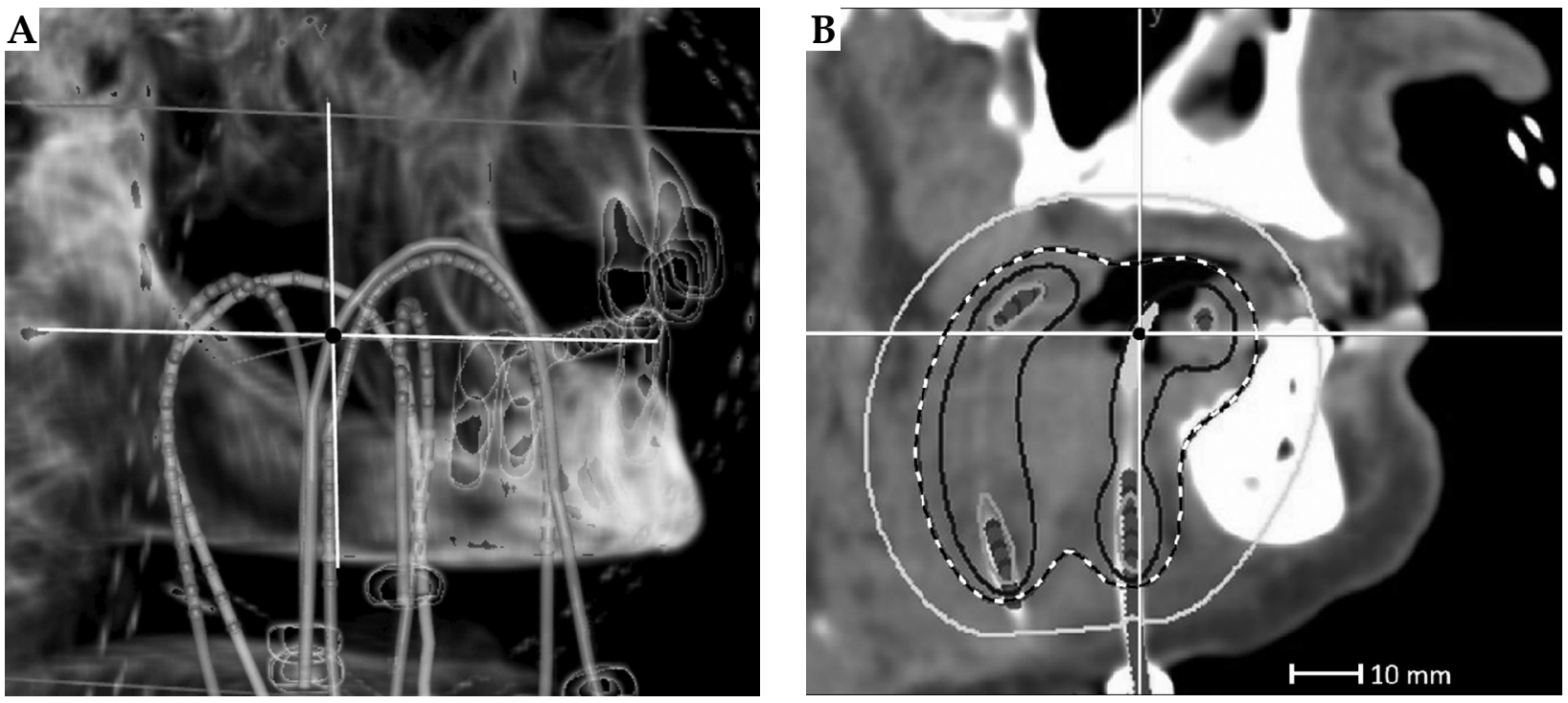

Fig. 3. Irradiation of the base of tongue for head and neck patient. A) Position of the applicators, origin of the coordinate system shows the location of the MOSFET detector. B) Isodose lines: light grey 50\%; black/white dashed: 100\%; black: 150\%; grey: $400 \%$ with the location of the MOSFET detector in the origin of coordinate cross

(Figure 4). The last measurement was in the $14^{\text {th }}$ fraction on the last day of treatment. We measured $4.7 \mathrm{~Gy}$ and it revealed a deviation of only $8.4 \%$ larger than predicted.

\section{Breast}

Results of the measurements in this group of patients demonstrated the necessity to divide the result analysis into 3 areas, orientated to the proximity of the position of the MOSFET detector in relation to the PTV. Figure 5 shows the deviations between the calculated and measured dose in a total of 62 measurements. The analysis ran as follows: First, dose values measured at the position of the tubes close to the ribs and close to surface showed deviations less than 1.0 Gy compared to the set point.

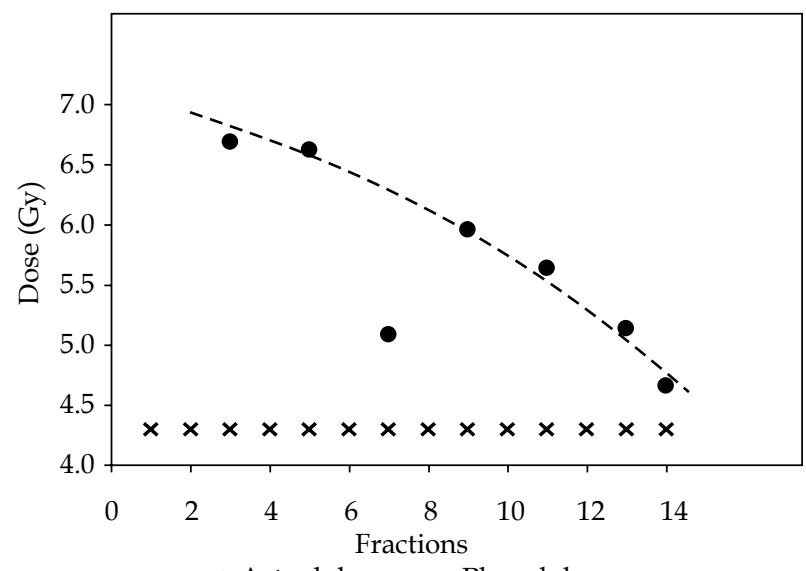

- Actual dose $\quad \times$ Planed dose

Fig. 4. Results of the measurement from the head and neck patient. The dose in the unit Gray is shown as a function of fraction number. Only measurement during the $7^{\text {th }}$ fraction is outside the observed range, probably due to wrong positioning of the detector inside the tube. The dashed line is only for guiding the eyes
The measured dose values presented a good agreement with the calculated data. It was noticeable that in these outcomes, the detector was always placed at the boarder of the PTV distant from the source positions. The closest dwell position to the detector was $9.2 \pm 0.1 \mathrm{~mm}$. Second, deviations between -0.8 Gy and 1.8 Gy compared to the set point were recorded at distances close to the PTV. The distance from the detector to the closest dwell position was $6.9 \pm 0.15 \mathrm{~mm}$. Third, the largest differences appeared inside the PTV and under the PTV. In this case, locations of detectors were set very close to or even inside the PTV. The deviations between $-0.5 \mathrm{~Gy}$ and 0.8 Gy compared to the set point could be found in $50 \%$ of all measurements. In 35\% of measurements, the dose values showed higher deviations than $1.0 \mathrm{~Gy}$ and in $15 \%$, the deviations were higher than 3.0 Gy. The average in the distances from the detector to the closest dwell position was $5.5 \mathrm{~mm}$. The number of tube applicators per

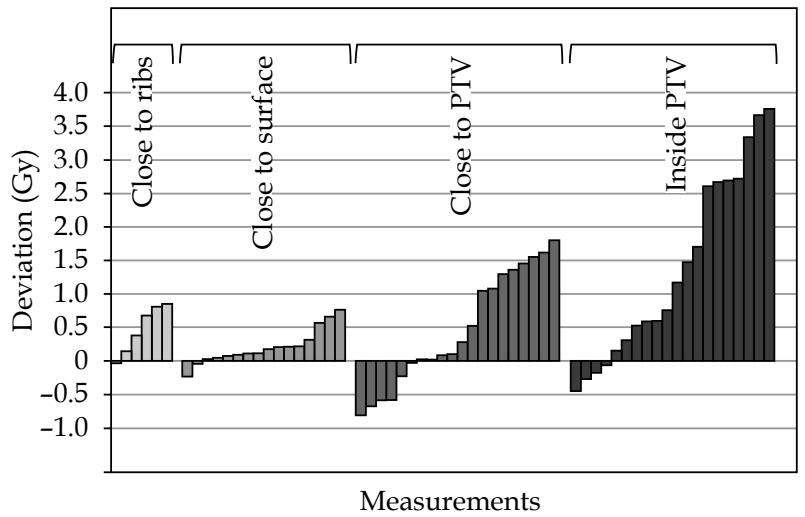

Fig. 5. Results of the 62 measurements from 10 patients with breast 270 cancer. The dose deviations in Gray are divided into areas, which are oriented to the proximity of the location of the MOSFET detector in relation to the PTV 
milliliter implanted inside the PTV (needle density) was considered to estimate the magnitude of possibility of disturbance inside the tissues. One breast cancer patient had the largest number of applicators per milliliters, 0.22 needles per milliliter in a PTV of $63.7 \mathrm{ml}$ and with $14 \mathrm{im}-$ planted applicators. The detectors were located inside the PTV, with an implanted geometry of three levels. In this case, it could be recognized as the greatest deviation between measured and calculated dose values compared to all included breast cancer patients. In deviations less than $0.5 \mathrm{~Gy}$, the average of the needle density was 0.18 needles per milliliter. In the deviations between $0.5 \mathrm{~Gy}$ and $1 \mathrm{~Gy}$, the average of the needle density was 0.16 needles per milliliter. In the deviation greater than $1 \mathrm{~Gy}$, the average of the needle density was 0.18 needles per milliliter.

\section{Discussion}

For the first time, the usefulness of MOSFET dosimetry is demonstrated in different cases of cancer irradiated with brachytherapy outside the field of prostate cancer. The accuracy of placing the source in a predicted location inside the tube has to be discussed. Since the outer diameter of the tube is $2 \mathrm{~mm}$ (6-French) and the inner diameter is $1.5 \mathrm{~mm}$, the source with a diameter of $0.86 \mathrm{~mm}$ results in a scope of $0.32 \mathrm{~mm}$ inside the tube to each side wall. This implies the possibility of displacement of the source up to $\pm 0.32 \mathrm{~mm}$ inside and perpendicular to the axis of the tube for each fraction. In addition, the tube location might change within the tissue in all three spatial directions. All these factors may contribute to minor changes in distances between the source dwell positions and MOSFET detector locations during treatment course. Therefore, it is likely that the source is located at each fraction in a slightly different position. The MOSFET detector has a very small sensitive volume for measurement, therefore suitable for accurate dosimetry for radioactive sources with a high-dose gradient. Measurement uncertainties described here show that it is impossible to record any statement about disturbances inside the tissue, if the detector is located closer than $5 \mathrm{~mm}$ to the nearest dwell position [10]. The temperature inside the body will not be higher than $40^{\circ} \mathrm{C}$ and should not have to be considered in this case [11].

\section{Thorax-wall}

The results in tube no. 3 were taken precisely between the two tubes with proximity to the respective points of $8.0 \mathrm{~mm}$ and $9.8 \mathrm{~mm}$. The results lie within the scope of the measurement accuracy.

In contrast, the results of the measured dose values in tube no. 6 are $15 \%$ higher as predicted; this corresponds to a position error of the detector $0.49 \mathrm{~mm}$ closer to the dwell position. This could have been caused by displacement of the source within the applicator tube up to a distance of $0.32 \mathrm{~mm}$ inaccuracy. Discrepancies in measurements close to the source could be attributed to a systematic error in the response of the MOSFET resulting from maximal dose gradient within very short distance [7]. Due to the inaccuracy of the measured value, it is not possible to issue a solid conclusion regarding the contribution of changes within the implanted tissues as edema and tissue inflammation.

\section{Head and neck}

In this case, the CT data were recorded immediately post-implant and it showed a deviation, which could not only be justified by the location uncertainties of the MOSFET detector or the source itself. Due to the possibility of longitudinal displacement of the MOSFET inside the tube $( \pm 1 \mathrm{~mm})$, there is a corresponding error in the measurement volumes to $\pm 0.2 \mathrm{~Gy}(4.65 \%)$.

The tubes were implanted in the form of a loop. During the treatment, the source moves within the curvature of the loop. Due to particular physical conditions, the position is always at the outer side of the curve, where the source always moves within the maximum distance to the inner side of the tube resulting in $0.32 \mathrm{~mm}$ difference. In that way, the distance to the measuring point rises to $4.22 \mathrm{~mm}$. Therefore, the distance between the detector and the dwell positions of the source seems to be large enough to obtain a correct measured dose value and the actual measurements also confirmed this. In the first measurement taken four days after surgery and CT scan, the dose value was $55.6 \%$ higher than predicted. The reason for the large discrepancies between the TPS calculated values and the MOSFET measured values is the formation of an edema, which reduces the distances between the tubes, especially the dwell positions. To measure such a high-dose, the detector position should be $1.7 \mathrm{~mm}$ distant from the source position and not $3.9 \mathrm{~mm}$ as it was planned. Furthermore, the measured dose could also be affected in all locations of the predetermined dwell positions by changes in distance caused by i.e. a tissue edema. The deviations decreased at the end of the treatment, which might be related to the resolution of the tissue edema following implantation procedure. Hereby, the distances between the tubes become at first smaller, as it is not possible to keep the distances constant during the tissue expansion because the jawbones build a limitation of the treated area. During the treatment time, the edema reduced its size and 12 days after recording the CT-data, the situation inside the tissue correlates to the condition right after procedure.

\section{Breast}

The dependence of the location on the determined dose values in terms of the proximity to PTV becomes very clear in these measurements. By placing the detector away from the PTV, the detectors are not surrounded by other tube applicators and show good agreement with predicted dose values. As a result, inflammation or edema occurring in the treated tissue would not affect the dose. The tubes kept their distances and would not move closer together. Close to the PTV, the deviation correlates to the proximity of the dwell positions to the detector. This can be recognized in an increase of the measured dose values compared to the calculated data. The largest deviations are found with the detector locations inside or under the PTV. The same pattern of results is noticed in 
all measurements regardless of the location of the measuring point, which could be explained by detumescence of the edema and subsequent decrease in distances between the tube applicators. After regression of the edema, all the dwell positions of the source are closer than in the treatment plan based on the CT-dataset taken one day following the implantation and after the development of the edema. The increased dose observed from the measurements from detector positions lying under the PVT could be a result of gravitation. CT dataset was recorded while the patients were lying flat, but the patients were treated in a sitting position. The actual measured dose was $56 \%$ more than the calculated dose by the TPS.

Due to the change in patient positioning, the gravitation led to a closer proximity of the applicator tubes, which could result in a higher measured dose compared to the calculated dose. To achieve the planned dose distribution, it is important to maintain the same positioning of the patient as it was during the recording of the CT dataset. The needle density does not seem to maintain a clear relationship with the formation of an edema. The disturbance in the tissue of the patient depends on the individual reaction in the special case.

\section{Conclusions}

Several factors should be considered during the use of in vivo dosimetry for quality assurance during the interstitial HDR brachytherapy. The timing of CT dataset acquisition in relation to the time of implantation procedure, the treated body region with its different implantation geometry of the applicators, and the individual nature of the treated organ are all contributing factors in the final results of in vivo dosimetry. The proximity to the PTV concerns the dose values due to the appearance of edema and the reproducibility of the patient's position during data recording as well as treatment affects the outcome of the dose distribution.

The conclusion emerges that the TPS calculated dose distribution and the measured dose values match best when interval time between implantation and recording of the CT is long. In this case, the edema has already disappeared before CT dataset obtaining and in this way, the planning fits to the anatomy.

\section{Disclosure}

The authors report no conflict of interest.

\section{References}

1. Schultze J, Schneider R, Kimmig B et al. Interstitial brachytherapy in head and neck region: when has imaging for treatment planning to be performed? Rontgenpraxis 2002; 54 : 232-237.

2. Chassagne D, Dutreix A, Ash D et al. Dose and Volume Specification for Reporting Interstitial Therapy. ICRU Report \#58, 1997.

3. Tanderup K, Beddar S, Andersen CE et al. In vivo dosimetry in brachytherapy. Med Phys 2013; 40: 070902.

4. Tremblay C, Gingras L, Archambault L et al. Characterization and use of MOSFET as in vivo dosimeters under ${ }^{192}$ Ir-irradiation for real-time quality assurance. Med Phys 2005; 32: 2003.
5. Qi ZY, Deng XW, Cao XP et al. A real time in vivo dosimetric verification method for high-dose-rate intracavitary brachytherapy of nasopharyngeal carcinoma. Med Phys 2012; 39: 6757-6763.

6. Cygler J. ESTRO IVD Workshop, Brussels, 5 December 2014.

7. Toye WC, Das KR, Todd SP et al. An experimental MOSFET approach to characterize 192Ir HDR source anisotropy. Phys Med Biol 2007; 52: 5329-5339.

8. Best Medical Canada. Mobile MOSFET On-line Wireless Dosimetry. Available at: http://www.mosfet.ca/product_mobilemos.html

9. Zilio VO, Joneja OP, Popowski Y et al. Absolute depth-doserate measurements for a 192Ir HDR brachytherapy source in water using MOSFET detectors. Med Phys 2006; 33: 1532-1539.

10. Cygler J, Scalchi P. MOSFET dosimetry in radiotherapy. AAPM Summer School, 2009.

11. Ehringfeld C, Schmid S, Poljanc K et al. Application of commercial MOSFET detectors for in vivo dosimetry in the therapeutic x-ray range from $80 \mathrm{kV}$ to $250 \mathrm{kV}$. Phys Med Biol 2005; 50: 289-303. 